\title{
Immunisation registers in Canada: progress made, current situation, and challenges for the future
}

J A Laroche (julie.a.laroche@phac-aspc.gc.ca) ${ }^{1}$, A J Diniz $^{1}$

1. Centre for Immunization and Respiratory Infectious Diseases, Public Health Agency of Canada, Ottawa, Ontario, Canada

Citation style for this article:

Laroche JA, Diniz AJ. Immunisation registers in Canada: progress made, current situation, and challenges for the future. Euro Surveill. $2012 ; 17(17)$ :pii=20158.

Available online: http://www.eurosurveillance.org/ViewArticle.aspx?Articleld=20158

Immunisation registers have the capacity to capture data on the administration of vaccine doses at the individual level within the population and represent an important tool in assessing immunisation coverage and vaccine uptake. In 1999, the National Advisory Committee on Immunization recommended that a network of immunisation registers be established in Canada. The Canadian Immunization Registry Network (CIRN) was established to coordinate the development of standards and facilitate the sharing of knowledge and experience to develop a national network of such registers. In 2003, the National Immunization Strategy identified immunisation registers as an important component in improving national immunisation surveillance. In addition, there has been consistent public and professional interest in a national immunisation register being available and considerable progress has been made in developing technologies to facilitate the capture of immunisation-related data. More specifically, the automated identification of vaccines, through the use of barcodes on vaccines, will facilitate collection of data related to administered vaccine doses. Nevertheless, challenges remain in the implementation of immunisation registers in all Canadian provinces and territories such that Canada still does not currently have a fully functional network of immunisation registers with the capacity to be interoperable between jurisdictions and to allow for data to be captured at the national level.

\section{Introduction}

In Canada, several millions doses of vaccines are administered every year. According to the immunisation schedules recommended by the National Advisory Committee on Immunization (NACI), the national committee tasked with making scientific recommendations on the use of vaccines in Canada, a child will receive 18 vaccinations to protect against 13 diseases by the age of two years, and 26 vaccinations by the age of 19 years [1]. These figures do not include seasonal influenza immunisation whereby the seasonal influenza vaccine may be received on an annual basis. In addition, several vaccines may be administered during a single immunisation visit. During each immunisation event, a healthcare provider must manually record details of the immunisation event in the patient's health record as well as in the patient's personal immunisation card or hand-held immunisation record.

Thus, information related to each immunisation event should become part of an individual's permanent health record. Access to this information is critical in the event of a vaccine recall, vaccine failure or of suspected adverse event following an immunisation (AEFI). Hand-held immunisation records can contain information such as antigen, brand name of vaccine, dose, date of immunisation, vaccine lot number, expiry date, route of administration, and injection site. Unfortunately, surveys conducted across Canada have shown that as many as $30 \%$ of parents have misplaced their child's immunisation record by the time their child is seven years old, $15 \%$ of immunisation records are incomplete, and $24 \%$ contain data entry errors [2]. Incomplete immunisation records result in up to $10 \%$ of the population being re-immunised needlessly, delays in the appropriate follow-up of AEFI, and vaccine supply issues. These issues result in increased costs to the health system and may potentially result in adverse health outcomes for vaccine recipients.

Reliable immunisation records at the individual level are essential to ensure that immunisations are provided according to the recommended immunisation schedules such that optimal protection against vaccine-preventable diseases (VPD) can result from immunisation [3]. At the population level, reliable and complete data from immunisation records are necessary for the assessment of vaccine uptake and coverage, vaccine effectiveness [4] and vaccine safety. Accurate and readily accessible immunisation records are also crucial in the event of a lot recall or vaccine failure because they can provide information necessary to track down potentially affected individuals.

Immunisation registers are information or software applications that have the capacity to perform the scheduling of immunisation appointments, the management and recording of immunisation events, notify 
when immunisations are due. These functions of immunisation registers allow them to serve as a tool to assess immunisation coverage. These populationbased databases have the potential ability, depending on the specific system used, to accurately assess, in real time, vaccine uptake at the national and regional levels, and personal immunisation status for individuals residing within a jurisdiction. Immunisation registers can also assist with the timely reporting of vaccine coverage, assessment of vaccine supply, identifying populations with low coverage, monitoring immunisation programmes designed to achieve specific target immunisation rates [5], as well as generating reminders to patients and recalls for immunisation visits. As a result, immunisation registers are considered to be one of the most effective strategies for improving coverage irrespective of provider [6]. Immunisation registers can also provide basic data to conduct vaccine effectiveness studies [7-9] as well as contribute to monitoring existing and new immunisation programmes. A number of countries have been successful in building and utilizing national population-based immunisation registries [4]. The Australian Childhood Immunization Register (ACIR) was the first complete national immunisation register and has been operational since 1996 [10].

The usefulness and power of a population-based immunisation register depend on the quality and quantity of the information it contains $[8,9]$. Keeping the records up-to-date and ensuring comprehensive use by all providers is important to warrant accurate projections for immunisation eligibility, vaccine supply and assessment of uptake. In some settings such as in Australia, monetary incentives have been supplied to providers for entering their patient immunisation data and for using immunisation registers to monitor their patient immunisation history and background [11].

In 1999, $\mathrm{NACl}$ recognised the importance of reliable, accessible, and standardised electronic immunisation records by passing a resolution recommending that a network of immunisation registers be established across Canada. A network of immunisation registers from the jurisdictions was proposed instead of a national immunisation register due to the fact that immunisation programme delivery is a provincial/territorial mandate in Canada. The goal of this resolution was to facilitate the maintenance of accurate immunisation records and the improvement of the management of vaccine supply.

The Canadian Immunization Registry Network (CIRN) was established to coordinate the development of standards and facilitate the sharing of knowledge and experience to develop a national network of immunisation registers [12]. CIRN is a Canadian immunisation committee working group made up of immunisation programme experts from all 13 Canadian provinces and territories involved in the development of immunisation registers as well as monitoring vaccine uptake in their respective jurisdictions. While CIRN members from the provinces and territories volunteer their time to the working group, the secretariat for CIRN is currently housed in the Centre for Immunization and Respiratory Infectious Diseases at the Public Health Agency of Canada (PHAC) where employees provide support to the working group. In addition, the working group has two co-chairs; a provincial/territorial cochair and a federal co-chair. The mandate of CIRN is to guide the provinces and territories in the development of a national network of compatible immunisation registries. CIRN works with Canadian provinces and territories to develop and agree upon national standards for immunisation registers as well as immunisation coverage assessment and provides input and expertise in the development of the national immunisation coverage surveys.

\section{Immunisation registers in Canada}

The 2003 National Immunization Strategy (NIS) identified the importance of coordinating common approaches to immunisation registers. One of the five key components of the NIS was to 'improve national surveillance and the transfer of (and access to) individual immunisation records, by establishing and maintaining a comprehensive, compatible national immunization registry network' [13]. Also in 2003, during the follow-up to the Severe Acute Respiratory Syndrome (SARS) outbreak in Canada, the 'Naylor Report' recommended CAD 100 million (approximately EUR 77 million) 'in incremental federal funding on new vaccines as well as improving the information systems to ensure that Canada meets an articulated health goal (and international norms) as regards vaccination coverage' [14].

In March 2004, responding to the lessons learned from the SARS outbreak, the federal government tasked Infoway with the development, in partnership with the Canadian provinces and territories, of a countrywide public health surveillance system. Infoway is a not-for-profit organisation created and funded by the federal government to accelerate the use of electronic health records (EHRs) in Canada through collaboration with the Canadian provinces and territories, healthcare providers and technology solution providers [15]. While CIRN and Infoway are two separate entities, the two groups are currently collaborating by the participation of some CIRN members in Infoway's Standards Collaborative Working Group [16] in developing agreedupon standards to be used in EHRs. The public health surveillance system developed through the partnership with Infoway was eventually called Panorama. Initially, a CAD 100 million (approximately EUR 77 million) fund was provided to support application software development. This funding initiative required the Canadian provinces and territories to provide the resources for training, equipment and implementation of the surveillance system. In 2008, an additional allotment of CAD 100 million was provided to advance the development and the implementation of Panorama [17]. Initially, Panorama had seven modules which were to be developed using existing commercially-available software 
applications, and which could be modified to respond to specific provincial and territorial needs. Two of the modules, the immunisation management and inventory management modules, were envisioned to provide the basis for a national network of immunisation registers. These modules were to be developed to include nationally agreed-upon functional and data standards. Moreover, the modules were to eventually provide each jurisdiction access to a standardised electronic immunisation register and an inventory management system to better manage immunisation events and vaccine supply, and assess immunisation coverage in their jurisdictions, as well as report standardised vaccine uptake data nationally. Panorama allows healthcare workers to view the immunisation history for individuals and particular groups in the population. In addition, lists of clients who are eligible and overdue for immunisation can be displayed by antigen, demographic or particular risk factors.

While the original scope of Panorama was to include, among other things, a Pan-Canadian approach to immunisation registers, some individual provinces and territories have developed and are currently using other immunisation register systems. These systems vary between jurisdictions in their availability to immunisation providers as well as to the extent of the data which they capture. Variability also exists between jurisdictions in validation procedures for immunisation register data as well as for the specific information contained in the register. For example, the currently available registers differ between jurisdictions in terms of the age groups for which information is captured. While data linkage between immunisation registers and clinical outcome databases is highly desirable, the capacity to link immunisation status to clinical outcome remains an issue in some contexts.

\section{Role of the federal government in immunisation registers}

Although Canadian provinces and territories are responsible for immunisation programme delivery and for implementing immunisation registers within their jurisdiction, the federal government is considered to provide leadership to the development of a national network of immunisation registers across Canada. Through NIS and CIRN, the federal government has provided leadership and coordination by supporting the development of national data and functional standards to guide immunisation register development and technologies such as the automated identification of vaccines. Automated identification of vaccines, or the use of scanners and barcodes on vaccine products, enhances considerably the quality and accuracy of the data captured in electronic registers by reducing the amount of time required by immunisation providers to create and maintain immunisation records and also reduces the possibility of errors related to data entry. The federal government has also contributed funds for the development of Panorama.

\section{Vaccine coverage assessment in Canada}

As is the case for other countries, Canada reports national vaccine coverage information to the World Health Organization. However, in order to compensate for the lack of a national network of immunisation registers to facilitate the accurate assessment of vaccine uptake, the PHAC currently uses telephone surveys to assess immunisation coverage in the Canadian population. The Childhood and Adult National Immunization Coverage Surveys are conducted approximately every two years to assess routine childhood immunisations and adult selected vaccines $[18,19]$. Cross-sectional vaccine coverage data are obtained for a selected set of age milestones for children and target groups for adults. Results from these surveys are used to monitor progress towards national targets, to report immunisation coverage estimates to international organisations, to improve planning for pandemic influenza, and to develop appropriately-targeted public education strategies.

These surveys are conducted using random-digit dialling or pre-existing sampling frames to contact households where eligible respondents might reside. Among the drawbacks associated with this current methodology are the facts that it is expensive and results in small sample sizes which do not permit for provincial and territorial vaccine coverage estimates, or the identification of under-served or under-immunised populations. The sample size selected for the national immunisation coverage surveys allows for immunisation coverage estimates with a $5 \%$ margin of error for each group. The use of surveys also precludes performing most studies related to immunisation programme effectiveness or evaluation. Finally, the use of telephone surveys introduces a responder bias as it is also becoming increasing difficult to recruit representative samples, due partly to the increase in the use of cell phones, and in the number of cell-phone-only households and changing lifestyles.

While national immunisation coverage estimates are obtained through surveys, provinces and territories use different methods to assess immunisation coverage within their jurisdiction depending on the availability of registers and other methods of collecting coverage data. To facilitate the collection of vaccine coverage data from the provinces and territories, PHAC and CIRN have developed national immunisation coverage reporting standards [20]. However, while these standards provide guidance to jurisdictions in reporting vaccine coverage, the standards have yet to be adopted. This is due to the fact that PHAC does not have the mandate to collect surveillance data from the jurisdictions. Thus, the adoption of a standardised methodology to estimate coverage remains problematic. Therefore, the PHAC will continue to implement national immunisation coverage surveys to estimate vaccine uptake nationally until a national network of immunisation registers is fully functional in all jurisdictions across Canada. 
Challenges to the implementation of immunisation registers across Canada

Despite overwhelming demand and clear support for a national network of immunisation registers [21,22], and approximately a decade after the $\mathrm{NACl}$ recommendation, only six of the 13 provinces and territories have developed centralised electronic immunisation registers that conform to national functional and data standards. Although Panorama was initially scheduled to roll out to the provinces and territories in 2009, it is substantially behind schedule and now will provide only a partial solution towards a national network of immunisation registers as some jurisdictions consider using other systems. While progress has been made, Panorama continues to face serious challenges due to cuts in scope and escalating costs leading to important delays. As a result, several provinces and territories have decided to opt out of using Panorama. The cuts in scope mean that several important agreed-upon national functional standards will not be included in Panorama. These include, but are not limited to, interoperability, or the ability to electronically share immunisation records between jurisdictions, and inclusion of automated identification technology.

\section{Public versus non-public immunisation providers}

In Canada, vaccines can be administered by immunisation providers that operate either in the public or in the non-public setting, depending on the jurisdiction where some provinces/territories deliver vaccines either through public health clinics or private physician's offices or a combination of both. The majority of immunisation registers in Canada currently capture information obtained only from public immunisation providers. These differences in immunisation delivery methods create considerable data completion issues in the larger Canadian provinces such as Ontario, Quebec, and British Columbia where physicians in private clinics administer most of the immunisations given in these jurisdictions.

\section{Acceptance of registers and 'knowledge, attitudes and beliefs' information}

The development, implementation and use of immunisation registers are well accepted amongst stakeholders and healthcare providers in Canada. However, beyond the development and implementation challenges previously discussed, and given the provincial and territorial mandate for immunisation programmes and delivery, data sharing agreements need to be developed and in place for jurisdictional level data to be shared with the federal government and included as part of national immunisation coverage estimates. Moreover, each province and territory needs to assess and deal with potential issues related to requirements regarding privacy for the use of such data before the implementation of an immunisation register or a public health surveillance system within a jurisdiction. While the use of immunisation registers to obtain coverage information represents an important tool to assess immunisation programmes, information on knowledge, attitudes, and behaviours/beliefs related to immunisation in the general population will need to be collected separately from coverage data obtained from immunisation registers thus making it impossible to assess the relationship between different knowledge, attitudes, behaviours/beliefs and vaccine uptake and to use this information to develop outreach and education programmes.

\section{Vaccine barcoding and automated identification technology}

To reduce the errors occurring from clinic staff manually entering vaccine name, lot number and expiry date into inventory and client records, $\mathrm{NACl}$ issued a recommendation in 1999 that barcodes be placed on all vaccine products manufactured in Canada to facilitate the automatic entry of scanned vaccine data [23]. A pilot project implemented with front-line immunisers in Alberta and Manitoba showed a $48 \%$ to $69 \%$ reduction in the time to record data and a $33 \%$ reduction in immunisation errors using peel-off, tagged and direct barcodes instead of manual entry [24]. In light of these findings and in support of NACl's recommendations, the Automated Identification of Vaccines Project Advisory Task Group (AIVP ATG) was founded, including representation from the vaccine and clinical software industries, healthcare professional organisations, and standard setting organisations [25].

In 2008, the AIVP ATG developed a five-year strategic plan. One of the first tasks was to perform an independent cost benefit analysis for the adoption and implementation of barcoding of vaccine products in Canada. Six different implementation options - varying in technical detail and the relative costs and benefits anticipated - were selected by the AIVP ATG for consideration. The study concluded that barcodes on vaccine products would be very beneficial and that these benefits would increase over time as technology advanced and new vaccines were introduced [26].

In 2009, AIVP ATG reached a consensus on vaccine barcode standards in Canada, including the placement of a Global Trade Identification Number (GTIN) - a unique product identifier - and lot number on primary packaging, with expiry date as an optional addition [25]. Canadian vaccine manufacturers have committed to adhering to these new standards over the next several years [25], and PHAC has developed the Vaccine Information Database System (VIDS), a web-based repository of information on all vaccines approved in Canada [27]. Vaccine manufacturers provide data (including GTIN, lot number, expiry date) for all of their products to the database (GS1) and transfer the data to PHAC, who is responsible for entering this information as well as lot number and expiry date into VIDS. Thus, when the barcode on a vaccine vial is scanned, the information is downloaded into the electronic immunisation or inventory record, eliminating the need for manual entry or paper-based recording. 
Vaccine manufacturers in Canada committed to voluntarily adopt the barcode standards and to include GTIN, lot number and expiry date on vaccine packages. By 2016, all vaccine products in Canada will be required to adhere to the agreed upon standards. Some vaccine labelling lines in Canada and the United States are currently able to print two-dimensional (2D) barcodes, and these vaccine products are shipped all over the world. Products manufactured in Europe will be the last to have the $2 \mathrm{D}$ barcodes as manufacturers have not yet agreed to the standards. A 2D barcode (such as the DataMatrix symbology from $\mathrm{GS}_{1}$, a barcode standard setting organisation) consists of printed squares or dots, spiralling outwards from the centre of the symbol. The 2D barcode includes a 14-digit Global Trade Identification Number (GTIN), expiry date and lot number [25]. Including the expiry date in the barcode is an optional labelling requirement, as the expiry date can be determined through lot number. Lot number and expiry date will continue to appear in human readable form on vaccine primary packaging as per Canadian labelling requirements.

The AIVP ATG is working with Canadian provinces and territories to encourage both public and private healthcare professionals to include barcoding in their software applications as well as to encourage the purchase scanners required to read barcodes. With the numerous different applications used in healthcare across the country, support to this community is essential in ensuring that barcodes on vaccine products are used to their fullest potential and that entering barcodes into immunisation registers results in reliable and timely immunisation and inventory records.

To support the use of barcodes in Canada, the AIVP ATG has committed to supporting early adopters of bar code technology, both in private and public healthcare settings, at point of vaccine administration as well as at the vaccine inventory level. Previous studies implementing barcode scanning on medications have employed linear barcodes containing a product identifier only [28-30]. However, placing a vaccine's GTIN and variable data (lot number and expiry date) on the limited space of a small vial requires a $2 \mathrm{D}$ matrix barcode.

To facilitate future adoption of barcode scanning technology, barcode readability and the incorporation of scanning the primary packaging into vaccination clinic workflow need to be examined. As the adoption of a new system can encounter user resistance, especially if it is unable to integrate into user workflow [31-32], it is important to understand how potential users perceive barcode scanning of vaccines and to identify aspects of the process requiring modification before its implementation in vaccination settings.

PHAC, in partnership with the PHAC/CIHR Influenza Research Network (PCRIN) and the Ontario Niagara Region, studied the integration of automated identification of vaccine products into inventory recording during seasonal influenza vaccination campaigns across Canada. Results demonstrated the readability of barcodes and positive user perceptions of this technology. While barcoding scanning was perceived to be beneficial in reducing errors, individual vial scanning for high volume clinics was found to be time consuming and may hinder adoption of this technology in these clinical settings [33]. These results highlight the importance of reviewing workflow processes and encouraging efficient practices specific to vaccination setting. . It is possible that the benefits of barcode scanning may be more apparent in settings where multiple vaccines and lot numbers are used [33].

Two early adoption studies are currently in the planning stages and will examine the use of barcode technology in a public or private health care setting where multiple vaccines are administered. A second study (in progress) examines the benefits of barcode technology in inventory management at the provincial depot level. Results from these studies are expected to be available and published in 2012.

\section{Conclusion}

Progress has been made in the last decade to develop a national network of immunisation registers. The federal government has contributed considerably through funds and leadership to this initiative. However, considerable challenges remain to the development and adoption of immunisation registers in all provinces and territories and even with the adoption of Panorama as a public health surveillance system or other immunisation registers, the vision of a national network of immunisation registers will not be realised unless innovative cost-efficient solutions are developed and the issue of interoperability between jurisdictions is resolved. Nevertheless, while several challenges need to be overcome for a fully operational network of immunisation registers to be available, several areas related to immunisation registers have made substantial progress. These include the development of standards and the automated identification of vaccine products through the barcoding of vaccines. While there have been challenges in the development of immunisation registers in Canada, other countries and regions with different immunisation surveillance systems in place such as in Europe have dealt with similar issues related to the need for homogeneous systems to provide comparable immunisation-related data across a region [34]. Nevertheless, other federated countries such Australia have been successful in setting up a national immunisation register and benefit from an operational national immunisation register to provide immunisation coverage data and vaccine safety data $[35,36]$. Finally, the upcoming years will most likely see greater developments in the availability of immunisation registers and in the accessibility of the relevant public health data. 


\section{Acknowledgments}

The authors would like to thank Teresa Mersereau, and Nashira Khalil for helpful discussions.

\section{References}

1. Public Health Agency of Canada (PHAC). Publicly funded Immunization Programs in Canada - Routine Schedule for Infants and Children including special programs and catch-up programs (as of December 2011). Ottawa: PHAC. 14 Dec 2011. Available from: http://www.phac-aspc.gc.ca/im/ptimprogprogimpt/table-1-eng.php

2. Public Health Agency of Canada (PHAC). Measuring up: results from the national immunization coverage survey, 2002. Canada Communicable Disease Report (CCDR). 2004:30. Available from: http://www.phac-aspc.gc.ca/publicat/ccdr-rmtc/04vol3o/ dr3005a-eng.php

3. Writing team for the Public Health Agency of Canada/Canadian Institutes of Health Research Influenza Research Network Vaccine Coverage Theme Group. Why collect individual-level vaccination data? CMAJ. 2010;182(3):273-5.

4. Salmon DA, Smith PJ, Navar AM, Pan WK, Omer SB, Singleton A et al. Measuring immunization coverage among preschoo children: past, present, and future opportunities. Epidemiol Rev. 2006;28:27-40.

5. Skull SA, Nolan TM. Australia needs an expanded immunisation register for further improvements in vaccine delivery and program evaluation. Med J Aust. 2007;187(9):504-5.

6. Jacobson VJ, Szilagyi P. Patient reminder and patient recall systems to improve immunization rates. Cochrane Database Syst Rev. 2005;(3):CDo0394.

7. Mahon BE, Shea KM, Dougherty NN, Loughlin AM. Implications for registry-based vaccine effectiveness studies from an evaluation of an immunization registry: A cross-sectional study. BMC Public Health. 2008;8:160.

8. Placzek H, Madoff LC. The use of immunization registrybased data in vaccine effectiveness studies. Vaccine. 2011;29(3):399-411.

9. Schauer SL, Maerz TR, Hurie MB, Gabor GW, Flynn JM, Davis JP. The use of an immunization information system to establish baseline childhood immunization rates and measure contract objectives. J Public Health Manag Pract. 2009;15(5):E6-12.

10. Hull BP, McIntyre PB, Heath TC, Sayer GP. Measuring immunisation coverage in Australia. A review of the Australian Childhood Immunisation Register. Aust Fam Physician. 1999;28(1):55-60.

11. Melbourne East GP Network. Increasing childhood immunization coverage rates in your practice. A Guide for Practice Staff. Melbourne East GP Network. Aug 2009. Available from: http://www.hhdgp.com.au/icms docs/82913 Practice_Data_Cleansing_Guide.pdf

12. Public Health Agency of Canada (PHAC). Canadian Immunization Registry Network (CIRN). Ottawa: PHAC. 10 Nov 2004; Available from: http://www.phac-aspc.gc.ca/im/cirnrcri/index-eng.php

13. F/P/T Advisory Committee on Population Health and Health Security (CPHHS). National Immunization Strategy: Final Report 2003. Canadian Ministry of Health: 2004. Available from: http://www.phac-aspc.gc.ca/publicat/nis-sni-03/pdf/ nat_imm_strat_e.pdf

14. Health Canada. Learning from SARS. Renewal of Public Health in Canada. A report of the National Advisory Committee on SARS and Public Health. Oct 2003. Available from: http://www. phac-aspc.gc.ca/publicat/sars-sras/pdf/sars-e.pdf

15. Canada Health Infoway [Internet]. Accessed 24 Apr 2012. Available from: https://www.infoway-inforoute.ca/lang-en/

16. Canada Health Infoway. Standards Collaborative Guide. Canada Health Infoway. [Accessed 24 Apr 2012]. Available from: https://www.infoway-inforoute.ca/flash/lang-en/scguide/ docs/StandardsCatalogue_en.pdf

17. Panorama. Pan-Canadian Public Health Communicable Disease Surveillance and Management Project. [Accessed 24 Apr 2012]. Available from: http://www-03.ibm.com/industries/ca/en/ healthcare/files/panorama_application_overview_final.pdf

18. Laroche J, Frescura A, Belzak L. Results from the 2006 and 2009 Childhood National Immunization Coverage Surveys. Can J Infect Dis Med Microbiol. 2010;21(4):205.

19. Laroche J, Frescura A, Belzak L. Results from the 2008 Adult National Immunization Coverage Survey. Can J Infect Dis Med Microbiol. 2010;21(4):204
20. Public Health Agency of Canada (PHAC). National Standards for Immunization Coverage Assessment: Recommendations from the Canadian Immunization Registry Network. Canada Communicable Disease Report. 2005;31(9). Available from: http://www.phac-aspc.gc.ca/publicat/ccdr-rmtc/05vol31/ dr3109a-eng.php

21. Eggertson L. Experts call for national immunization registry, coordinated schedules. CMAJ. 2011;183(3):E143-4.

22. Heidebrecht CL, Foisy J, Pereira JA, Quan SD, Willison DJ, Deeks $\mathrm{SL}$, et al. Perceptions of immunization information systems for collecting pandemic $\mathrm{H}_{1} \mathrm{~N}_{1}$ immunization data within Canada's public health community: A qualitative study. BMC Public Health. 2010;10:523.

23. Public Health Agency of Canada PHAC). Supplement. Canadian national report on immunization, 2006. Canadian Communicable Disease Report (CCDR). 2006;32S3. Nov 2006. Available from: http://www.phac-aspc.gc.ca/publicat/ccdrrmtc/o6vol32/32s3/index-eng.php

24. Public Health Agency of Canada (PHAC). Evaluation of the Automated Identification of Vaccine Pilot Project. Ottawa: PHAC. Sep 2005.

25. Public Health Agency of Canada (PHAC). Canadian consensus statement on proposed standards for barcodes on vaccine products. Ottawa: PHAC. 31 Aug 2010. Available from: http:// www.phac-aspc.gc.ca/im/bar-codes-eng.php

26. HDR Decision Economics. Cost Benefit Analysis for Adoption and Implementation of the Automated Identification (Bar Coding) of Vaccine Products. 6 Feb 2009.

27. Public Health Agency of Canada (PHAC). Vaccine Identification Database (VIDS). [Internet]. Accessed 24 Apr 2012. Available from: http://www.vids-siv.phac-aspc.gc.ca/login.php

28. DeYoung JL, VanderKooi ME, Barletta JF. Effect of barcodeassisted medication administration on medication error rates in an adult medical intensive care unit. Am J Health Syst Pharm. 2009;66(12):1110-5.

29. Morriss FH Jr, Abramowitz AW, Nielson SP, Milavetz G, Michael SL, Gordon SN, et al. Effectiveness of a barcode medication administration system in reducing preventable adverse drug events in a neonatal intensive care unit: A prospective cohort study. J Pediatr. 2009;154(3):363-8.

30. Paoletti RD, Suess TM, Lesko MG, Feroli AA, Kennel JA, Mahler JM, et al. Using barcode technology and medication observation methodology for safer medication administration. Am J Health Syst Pharm. 2007;64(5):536-43.

31. Schneider R, Bagby J, Carlson R. Barcode medication administration: A systems perspective. Am J Health Syst Pharm. 2008;65(23):2216-9.

32. Campbell EM, Sittig DF, Ash JS, Guappone KP, Dykstra $\mathrm{RH}$. Types of unintended consequences related to computerized provider order entry. J Am Med Inform Assoc. 2006;13(5):547-56.

33. Integrating Automated Identification of Vaccine Product (AIVP) into Inventory Recording in Influenza Vaccination Clinics Across Ontario. 2010. Available from: http://resources.cpha.ca/CPHA/ Conf/Data/2011/A11-630ae.pdf

34. Haverkate M, D’Ancona F, Johansen K, van der Velden K, Giesecke J, Lopalco PL. Assessing vaccination coverage in the European Union: is it still a challenge? Expert Rev Vaccines. 2011;10(8):1195-205.

35. Hull B, Dey A, Mahajan D, Campbell-Lloyd S, Menzies RI, McIntyre PB. NSW Annual Immunisation Coverage Report, 2009. N S W Public Health Bull. 2010;21(9-10):210-23.

36. Gold M, Dugdale S, Woodman RI, McCaul KA. Use of the Australian Childhood Immunisation Register for vaccine safety data linkage. Vaccine. 2010;28(26):4308-11. 